\title{
Europium chelate solid laser based on morphology-dependent resonances
}

\author{
Hiroshi Taniguchi \\ Department of Electrical and Electronic Engineering, Faculty of Engineering, Iwate University, 4-3-5 Ueda, \\ Morioka 020, Japan \\ Junji Kido \\ Department of Materials Science and Engineering, Faculty of Engineering, Yamagata University, \\ 4-3-16 Jonan, Yonezawa 992, Japan \\ Masahisa Nishiya and Shinobu Sasaki \\ Department of Electrical Engineering, Faculty of Engineering, Iwate University, 4-3-5 Ueda, Morioka 020, \\ Japan
}

(Received 27 March 1995; accepted for publication 14 June 1995)

We show lasing (with the threshold of $\mu \mathrm{J}$ order) due to morphology-dependent resonances (MDRs) from solid polystyrene spheres with volume-distributed europium (Eu) chelates. Lasing spectra depending on MDRs are reported, and a practical usefulness of the Eu chelate laser with the solid spheres is described compared with the previous one with liquid spheres.

In the beginning in 1960, the extremely intense fluorescence observed in some rare-earth chelates interested many workers in the laser field; however, the laser fields using chelates scarcely developed, after that time. This seems to be due to the need to operate with considerably high input energy and to the rather complex characteristics in the chelate chemical/solvent combinations.

Some investigations on rare-earth chelate solutions have reported laser operation, at about $-100^{\circ} \mathrm{C}$ and below, ${ }^{\prime}$ at room temperature $\left(25^{\circ} \mathrm{C}\right),{ }^{2}$ with lasing thresholds of about $1000 \mathrm{~J}$ or above in europium (Eu) chelates, and with low thresholds of about $120 \mathrm{~J}$ in neodymium (Nd) systems. ${ }^{3}$ In these previous studies, special precautions for laser-cavity construction (including pumping volumes), solvent, lasant concentration, and operating temperature, have been exercized in the experiments, because the chelates are strongly absorptive ${ }^{1}$ and oxidized to nonfluorescent products, ${ }^{3}$ for favorable fluorescent efficiency. Thus in previous chelate lasers, the required input energy for laser oscillation was as high as that for ruby lasers, in consequence, means to avoid overheating were needed.

On the other hand, recently many investigations on the light-scattering properties of dielectric spheres have interested many workers. The spherical resonator have been widely used because of the very high quality factor ( $Q$ value) of electromagnetic whispering-gallery mode ${ }^{4}$ or morphology-dependent resonances (MDRs) in the dielectric sphere with micrometer-sized droplets ${ }^{5,6}$ or solid particles. ${ }^{7,8}$ The high $Q$ value of spherical cavities has been shown to significantly lower the threshold for lasers. ${ }^{5,6}$ More recently, whereby the spherical Eu chelate laser (of liquids) with ultralow thresholds (of a $\mu \mathrm{J}$ order) has been achieved, ${ }^{9}$ however, in which the problems of uniform radiation in much higher concentrations encountered in the application have been suggested. In fact, the extremely increasing fluorescence has been observed as the chelate concentrations increase, by incoherent ultraviolet (UV) lamp illumination, in the preliminary work of Ref. 9. We consider that the nonuniform radiation in the liquid spherical cavity in the higher concentrations ${ }^{9}$ is dominantly due to the inhomogeneous dis- tribution of chelate molecules in the liquid solvent. Accordingly, we have devised a technique of making a spherical cavity, from liquid to solid, to remove the rather complex characteristics between ligands and solvents. ${ }^{2,10,11}$

In this letter, we report the first experimental achievement of Eu chelate solid spherical laser with ultralow thresholds (of a $\mu \mathrm{J}$ order, similar to previous Eu chelate liquid lasers based on MDRs ${ }^{9}$ ). In most previous studies of rareearth chelate lasers, ${ }^{1-3,9-11}$ liquid host media has been used except for one example of didymium chelate rods with solid hosts. ${ }^{3}$ The Eu chelate solid laser, which is free from the effects of solvents, may be one of the candidates for the practical use in chelate laser fields.

An experimental arrangement similar to those described previously ${ }^{9,12}$ is used. A transversely excited atmospheric (TEA) UV $\mathrm{N}_{2}$ laser ( $337.1 \mathrm{~nm}$ ) is used to pump a spherical Eu-chelate-distributed laser, focusing the $\mathrm{UV} \mathrm{N}_{2}$-laser pump beam with a spherical quartz lens (SQL) after beam shaping through a circular aperture. Spectra of light emitted from the laser or spatial images of light in the sphere are analyzed with an optical multichannel analyzer (OMA) or observed with a color charge coupled device (CCD) camera, respectively, mounted on a microscope.

A position of single Eu chelate spheres, having $\sim 20-70$ $\mu \mathrm{m}$ diameter for the $\sim 1 \mathrm{~nm}$ resolution of the OMA, which is on a glass slide under the microscope,${ }^{12}$ is adjusted finely in the vicinity of the focal point of the SQL with $5 \mathrm{~cm}$ focal length, in the microscope field. The focusing pump beam diameter was larger than the sphere diameter.

The volume-distributed spherical Eu chelate lasers (at 8 wt $\%$ of Eu chelates to polystyrene hosts) were prepared by synthetically dispersing $\mathrm{Eu}(\mathrm{DBM})_{3}(\mathrm{Phen})$ (DBM $=$ dibenzoylmethane, Phen $=$ phenanthroline $)^{9}$ in polystyrene spheres in our laboratory.

The single Eu-distributed sphere (Eu sphere) was pumped by the UV $\mathrm{N}_{2}$ laser, having a $1.3 \mathrm{~ns}$ full width at half-maximum and $3 \mathrm{~kW}$ peak power $(\sim 4 \mu \mathrm{J}$ pulse energy) at $337.1 \mathrm{~nm}$. By finely adjusting the position of the Eu sphere in the vicinity of the focal point of the SQL, it was observed by the CCD camera that the rim of the Eu sphere above 


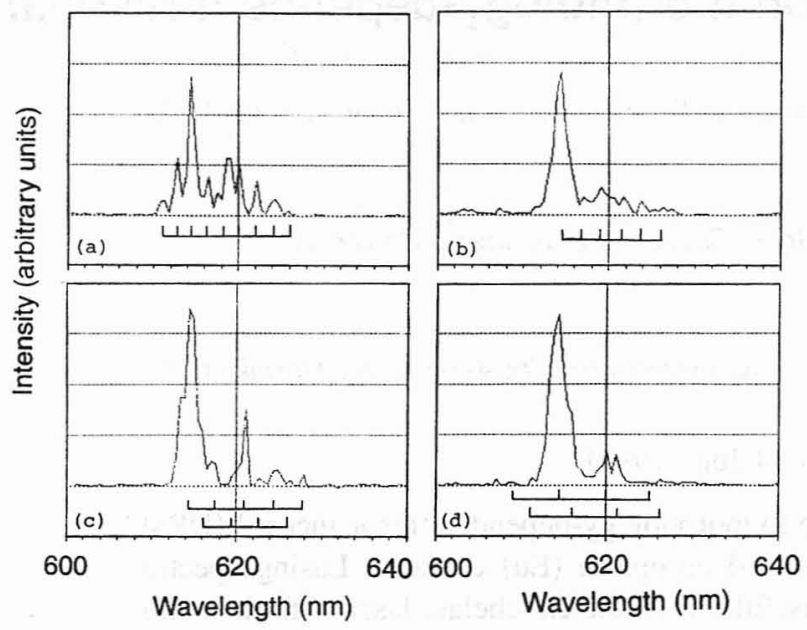

FIG. 1. MDR-lasing spectra from Eu-distributed solid spheres. Sphere diameter $D=50,40,30$, and $17 \mu \mathrm{m}$ in (a), (b), (c), and (d), respectively. The single [(a) and (b)] and double scales [(c) and (d)] below the each spectra exhibit the mode spacing expected from the formula due to MDRs (see Ref. 13 ), for the refractive index $n=1.59$ (for polystyrene).

threshold is brighter than the center, while the image of the emission pattern is darker and rather uniform below threshold, which is similar to those in previous studies, ${ }^{9,12}$ suggesting the features of MDR lasing with high $Q$ values. Thus the present Eu chelate solid lasers are similar to liquid ones in previous study, however, it is noted that the intensity of lasing emission in solid ones exhibits $\sim 100$ times enhancement over that in the previous liquid ones. Such findings suggest that more effective laser performance due to MDRs is possible in solid spheres than in liquid spheres. We believe, at present, that this is dominantly due to more homogeneous distribution of Eu chelates in the present solid spheres, different from the states in the liquid spheres described in the beginning of this letter.

To obtain experimental data on the sphere size dependence of MDR spacing in lasing, individual single spheres with different diameters were examined. Figure 1 shows the single-shot MDR-lasing spectra from the Eu-distributed sphere with various sphere size; sphere diameter $D=50,40$, 30, and $17 \mu \mathrm{m}$ in Figs. 1(a), 1(b), 1(c), and 1(d), respectively. Quasiperiodic structural peaks are seen in each spectra of Fig. 1. The equidistant scales exhibiting the mode spacing expected from the formula due to MDRs of spheres, ${ }^{13}$ which depends on sphere size and the fixed refractive index $n=1.59$ for polystyrene, are also attached below the each spectra. Figures 1(a) and 1(b) [with larger D] and Figs. 1(c), 1(d) [with smaller $D$ ] exhibit the single and double structural peaks, respectively, accompanied with the single and double scales below the each spectra corresponding to these structural peaks. The double structural peaks in Figs. 1(c) and 1(d), although there exists the limitation of the OMA resolution $(\sim 1 \mathrm{~nm})$, seem to be observed according to the separated TE or TM modes in the smaller spherical cavities. It is found in Fig. 1 that the Eu chelate solid laser also operates in nearly the same spectral region of $\mathrm{Eu}^{3+}$ (at $\sim 614 \mathrm{~nm}$ with the largest peak), as known that the Eu-doped solution system operates at $613 \mathrm{~nm}$ and $615 \mathrm{~nm}$ of the Eu-

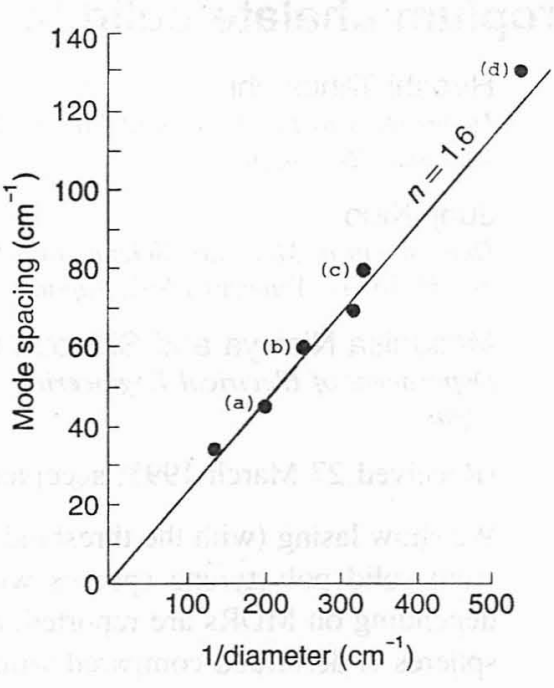

FIG. 2. Mode spacing in MDR lasing depending on sphere diameters. The straight line is obtained in the case of the refractive index being $n=1.6$ for the polystyrene spheres and the plots with (a)-(d) correspond to (a) $-(d)$ in Fig. 1, respectively.

benzoylacetonate $\left(\mathrm{EuB}_{3}\right),{ }^{1}$ and at $612 \mathrm{~nm}$ of Eu-benzoyltrifluoroacetonate $\left[\mathrm{Eu}\left(\mathrm{BTF}_{4}\right) \mathrm{P}\right],{ }^{2}$ arising from an electric dipole transition between the ${ }^{5} D_{0}$ and ${ }^{7} F_{2}$ levels (corresponding to $600-625 \mathrm{~nm}$ ) of $\mathrm{Eu}^{3+}$. This is also analogous to the Eu chelate liquid laser with the spherical cavity. ${ }^{9}$

Measured spectral data on the sphere size dependence of MDR spacing are shown in Fig. 2, in which the straight line is obtained in the case of the refractive index being $n=1.6$ for the polystyrene spheres and the plots with Figs. 2(a) -2 (d) corresponding to Figs. 1(a)-1(d), respectively. The measured mode spacing is proportional to the inverse diameter of the sphere, and this indicates that the present Eu chelate solid laser operates according to the MDRs in the spherical cavity.

We have presented the first experimental achievement of Eu chelate solid spherical laser with ultralow thresholds (of a $\mu \mathbf{J}$ order). The present laser with low thresholds may operate with high $Q$ value based on MDRs in the spherical cavity. In the resonator construction of the spherical cavity, only the small parts near the spherical surface serve essentially as the cavity. In this sense, the spherical cavity construction is the most favorable one for chelate lasers, which have strongly absorptive features. Furthermore, the present Eu chelate solid laser is free from the effects of solvents, the laser may be one of the candidates for practical use in chelate-laser fields.

The authors gratefully acknowledge the continuous encouragements from Professor H. Inaba, Tohoku Institute of Technology, Japan.

'A. Lempicki and H. Samelson, Phys. Lett. 4, 133 (1963).

${ }^{2}$ H. Samelson, A. Lempicki, C. Brecher, and V. Brophy, Appl. Phys. Lett. 5. 173 (1964).

${ }^{3}$ B. Whittaker, Nature 228, 157 (1970).

${ }^{4}$ C. G. B. Garrett, W. Kaiser, and W. L. Bond, Phys. Rev. 124, 1807 (1961).

${ }^{5}$ H.-M. Tzeng, K. F. Wall. M. B. Long, and R. K. Chang. Opt. Lett. 9, 499 (1984).

${ }^{6}$ H.-B. Lin, A. L. Huston, B. L. Justus. and A. J. Campillo, Opt. Lett. 11. 614 (1986). 
${ }^{7}$ R. E. Benner, P. W. Barber, J. F. Owen, and R. K. Chang, Phys. Rev. Lett. 44, 475 (1980).

${ }^{8}$ M. K. Gonokamı, K. Takeda, H. Yasuda, and K. Ema, Jpn. J. Appl. Phys. 31. L99 (1992).

${ }^{9}$ H. Taniguchi, H. Tomisawa, and J. Kido, Appl. Phys. Lett. 27, 1578 (1995).
${ }^{10}$ A. Lempicki, H. Samelson, and C. Brecher, Appl.Opt. Suppl. 2, 205 (1965).

${ }^{11}$ H. Samelson, C. Brecher, and A. Lempicki, J. Chim. Phys. 64. 165 (1967).

${ }^{12} \mathrm{H}$. Taniguchi and S. Tanosaki, J. Appl. Phys. 32, L1421 (1993).

${ }^{13}$ X.-X. Qian, J. B. Snow. H.-M. Tzeng, and R. K. Chang. Science 231. 486 (1986) 